\title{
Signals features extraction in liquid-gas flow measurements using gamma densitometry. Part 2: frequency domain
}

\author{
Robert Hanus ${ }^{1, a}$, Marcin Zych ${ }^{2}$, Leszek Petryka ${ }^{2}$, Marek Jaszczur ${ }^{2}$ and Paweł Hanus ${ }^{3}$ \\ ${ }^{1}$ Rzeszow University of Technology, 35-959 Rzeszow, Poland \\ ${ }^{2}$ A GH University of Science and Technology, 30-059 Krakow, Poland \\ ${ }^{3}$ Test \& Measurement Solutions sp. z o.o., 53-025 Wrocław, Poland
}

\begin{abstract}
Knowledge of the structure of a flow is really significant for the proper conduct a number of industrial processes. In this case a description of a two-phase flow regimes is possible by use of the time-series analysis e.g. in frequency domain. In this article the classical spectral analysis based on Fourier Transform (FT) and Short-Time Fourier Transform (STFT) were applied for analysis of signals obtained for water-air flow using gamma ray absorption. The presented method was illustrated by use data collected in experiments carried out on the laboratory hydraulic installation with a horizontal pipe of $4.5 \mathrm{~m}$ length and inner diameter of $30 \mathrm{~mm}$ equipped with two ${ }^{241} \mathrm{Am}$ radioactive sources and scintillation probes with $\mathrm{NaI}(\mathrm{Tl})$ crystals. Stochastic signals obtained from detectors for plug, bubble, and transitional plug - bubble flows were considered in this work. The recorded raw signals were analyzed and several features in the frequency domain were extracted using autospectral density function (ADF), cross-spectral density function (CSDF), and the STFT spectrogram. In result of a detail analysis it was found that the most promising to recognize of the flow structure are: maximum value of the CSDF magnitude, sum of the CSDF magnitudes in the selected frequency range, and the maximum value of the sum of selected amplitudes of STFT spectrogram.
\end{abstract}

\section{Introduction}

Knowledge of a two-phase flow structure is significant for the proper conduct a number of industrial processes, so flow regime identification inspires many studies. Recent works shows use for this purpose machine learning methods as e.g. artificial neural networks [1-12]. Generally computational intelligence methods utilize various features of signals in the time, frequency and state-space domain. Features of signals in the frequency domain may be determined using the Fourier Transform (FT), Short-Time Fourier Transform (STFT), wavelet transform and other methods [13-15].

In this work FT and STFT are applied to signals obtained in two-phase liquid-gas flow measurements using gamma-ray absorption, where data were collected in experiments carried out on a laboratory hydraulic installation with a horizontal pipe of $4.5 \mathrm{~m}$ length and inner diameter of $30 \mathrm{~mm}$. Stochastic signals obtained for three structures of air-water flow as plug, bubble, and transitional plug - bubble one were considered. Laboratory stand and gamma absorption equipment are presented in the first part of this work and and are described in detail in [16-19].

For such features of recorded signals comparison in the frequency domain as maximum value of the autospectral density function (ADF), maximum value of the cross-spectral density function (CSDF) magnitude, sum of the ADF and sum of the CSDF in the selected frequency range, and the maximum value of the sum of selected amplitudes of STFT spectrogram were extracted.

\section{Spectral analysis of signals using FT}

The time records of stochastic signals obtained from gamma absorption set were presented in the first part of this work. These signals after pre-processing were employed for the analysis in the frequency domain. The one-sided autospectral density function $G_{x x}(f)$, and onesided cross-spectral density function $G_{x y}(f)$ determined in practice for the frequency range $0<f<\infty$ are defined by the following formulas $[13,20]$ :

$$
\begin{aligned}
& G_{x x}(f)=2 \int_{-\infty}^{\infty} R_{x x}(\tau) e^{-j 2 \pi f \tau} d \tau \\
& \underline{G}_{x y}(f)=2 \int_{-\infty}^{\infty} R_{x y}(\tau) e^{-j 2 \pi f \tau} d \tau
\end{aligned}
$$

where: $R_{x x}(\tau)$, and $R_{x y}(\tau)$ are the autocorrelation and crosscorrelation function respectively, $f$ - frequency, $\tau$ - time delay.

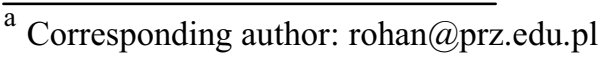


Table 1. ADF and $|\mathrm{CSDF}|$ of signals from the first scintillation probe obtained in BUB6, BUB 4 and BUB1 experiments.

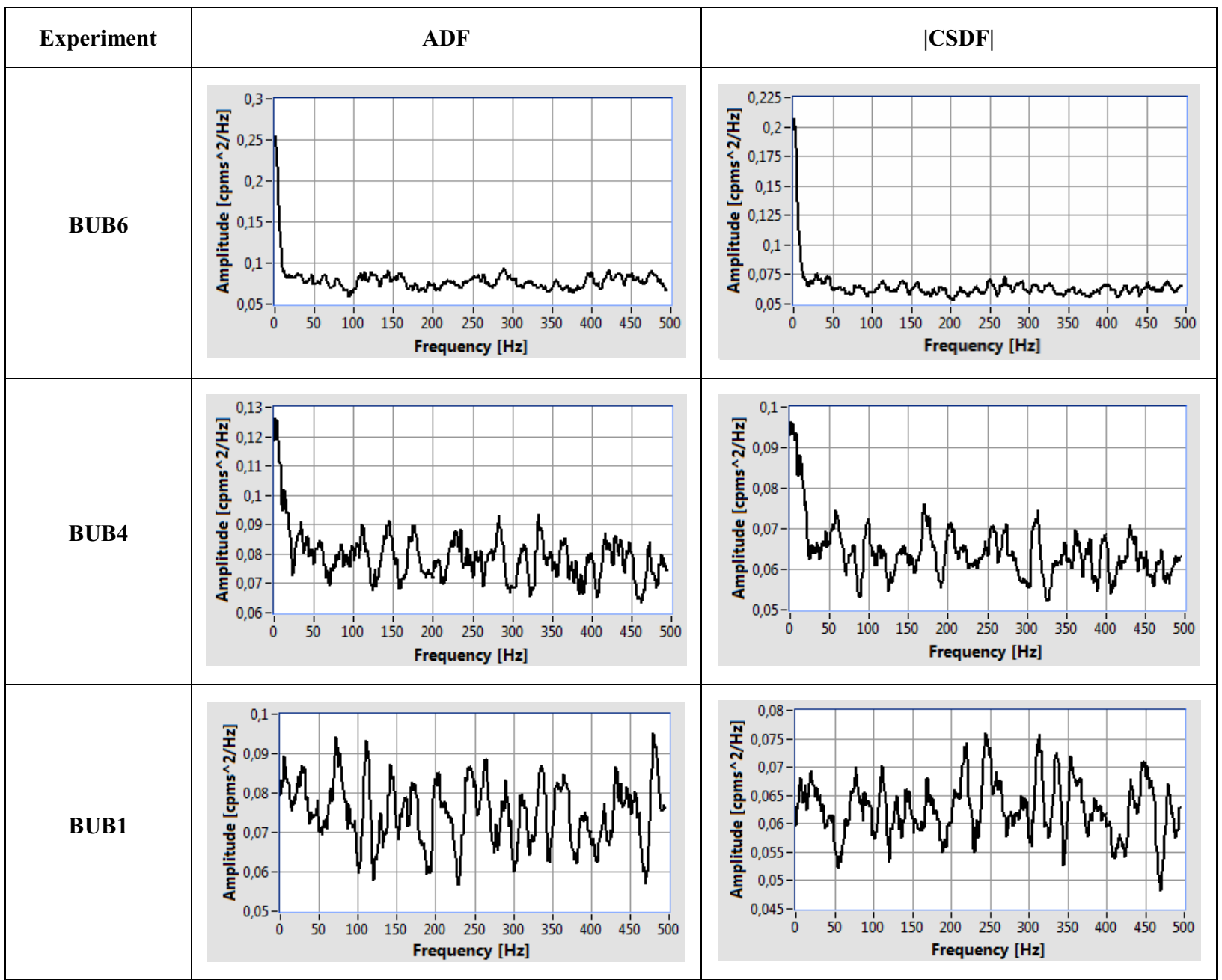

Since the cross-spectral density is a complex function, the module and phase of the CSDF are given as:

$$
\begin{gathered}
|C S D F|=\left\{\operatorname{Re}\left[G_{x y}(f)\right]^{2}+\operatorname{Im}\left[\underline{G}_{x y}(f)\right]^{2}\right\}^{0,5} \\
\arg (C S D F)=\operatorname{arctg}\left\{\operatorname{Im}\left[\underline{G}_{x y}(f)\right] / \operatorname{Re}\left[\underline{G}_{x y}(f)\right]\right\}
\end{gathered}
$$

In consequence the phase distributions of CSDF (4) were used to measure the average velocity of the dispersed phase of the two-phase flow, like a cross-correlation [20-26].

Additionally the spectral analysis can be used to recognize the type of the gas bubbles transportation in the flow. The literature shows a use for this purpose ADF [27], however, according to [19], better results can be accomplished using the magnitude of cross-spectral density (3).

Table 1 shows the ADF and $|\mathrm{CSDF}|$ determined for signals in experiments BUB6 (plug flow), BUB 4 (transitional plug-bubble flow) and BUB1 (bubble flow). Windowing, overlapping of data segments and combined ensemble-averaging and frequency-averaging were used to $\mathrm{ADF}$ and CSDF estimation [21].

Based on the collected experiments, the following three parameters ("features") of spectral densities ADF and $|\mathrm{CSDF}|$ were identified:

- maximum of spectral density,

- $\quad$ area under the graph of spectral density for the selected frequency range (experimentally selected range from $0 \mathrm{~Hz}$ to $f_{g}=20 \mathrm{~Hz}$ ).

- given above area for the selected frequency range related to the total area.

The graphs presented in figure 1 show the values of the descripting features as a function of the segment number. In the spectral analyses the following settings determined experimentally were used: segment width of 10,000 samples, Hamming window, 20 samples width of moving average window, $80 \%$ overlapping [21]. 
a)

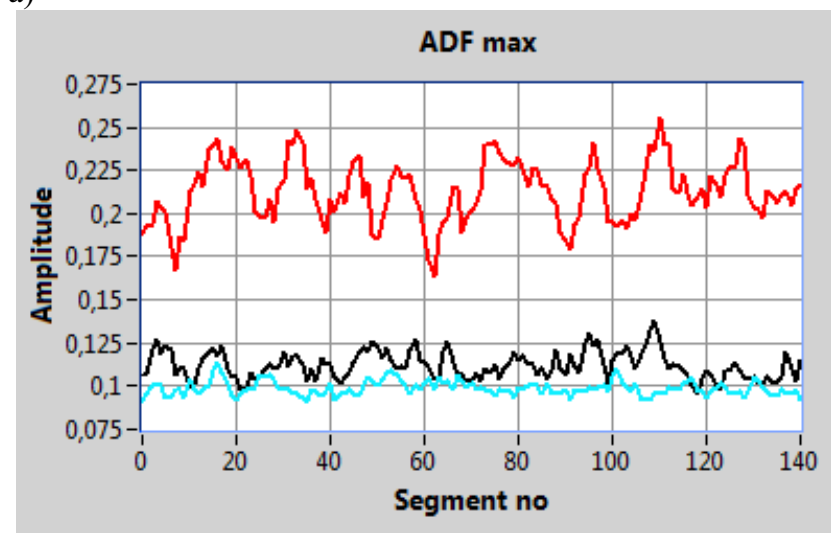

b)

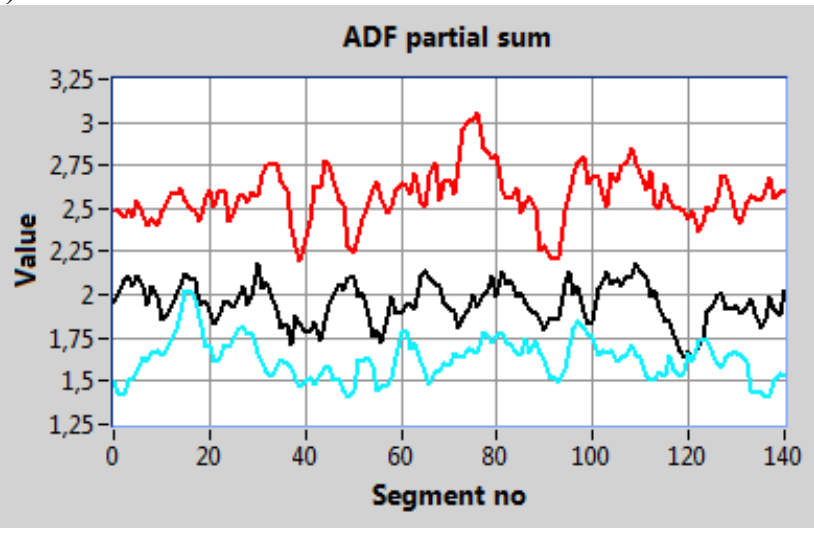

c)

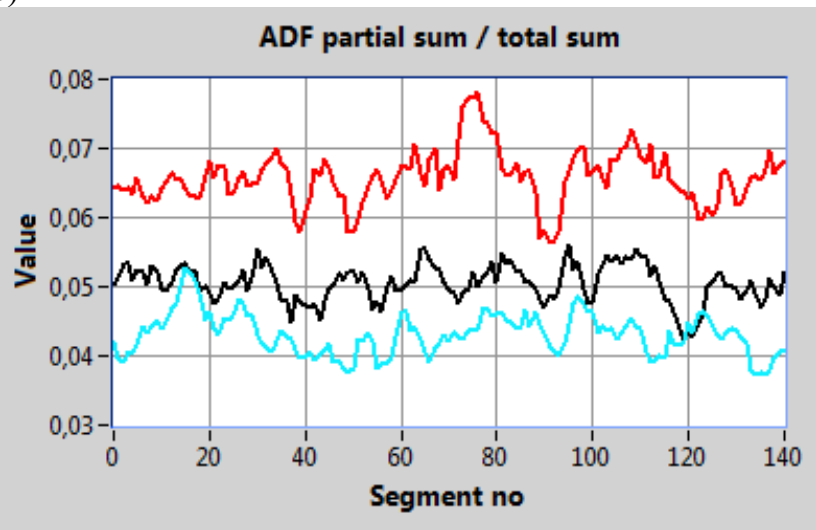

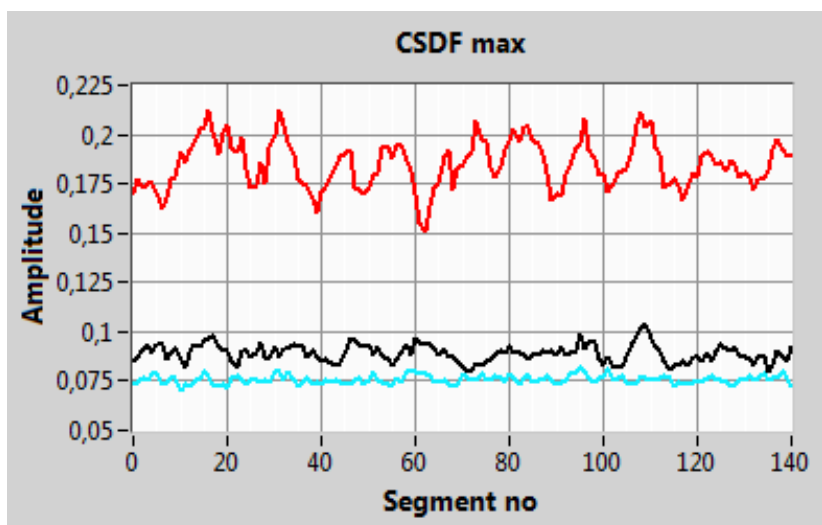
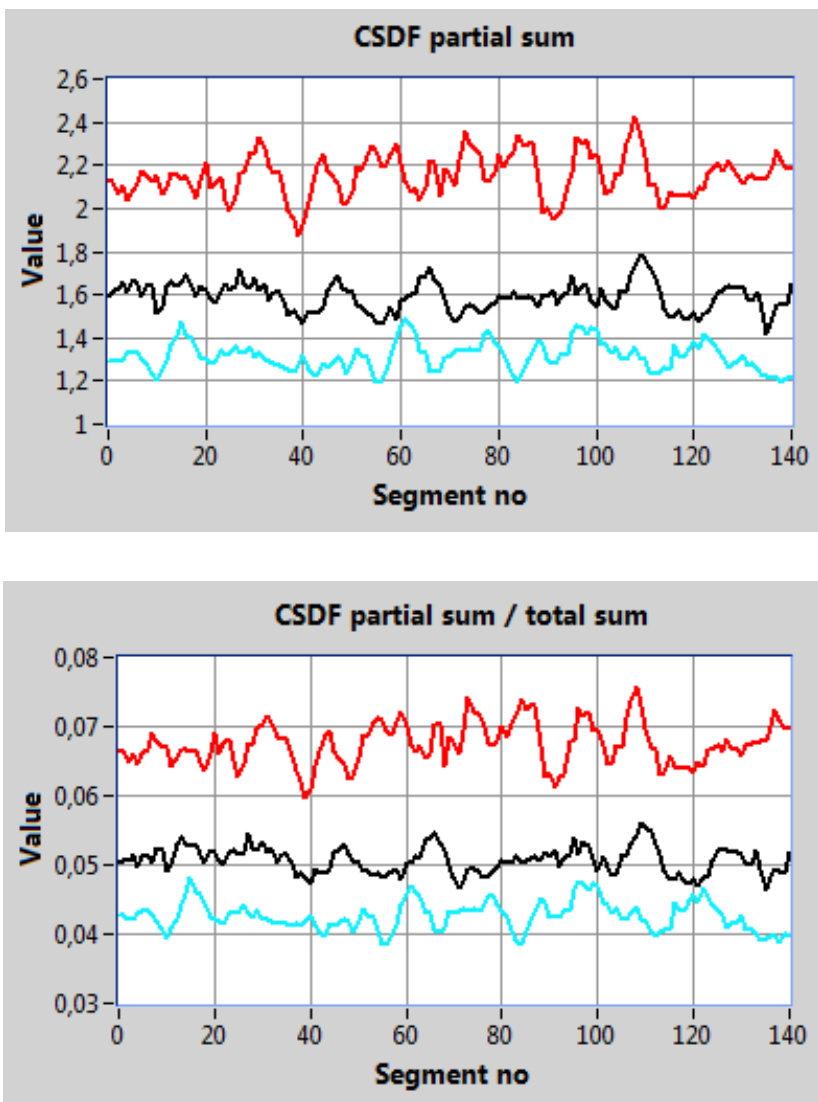

BUB601 - plug flow, BUB401 - transitional plug - bubble flow,

Figure 1. Features determined on the basis of ADF and $|\mathrm{CSDF}|$ : (a) maximum value of the ADF and $|\mathrm{CSDF}|$, (b) sum of the ADF and $|\mathrm{CSDF}|$ in the selected frequency range, (c) relative sum of the ADF and $|\mathrm{CSDF}|$

\section{The analysis of signals using STFT}

STFT is time-frequency analysis method in which the signal $x(t)$ is divided into short, consecutive, possibly overlapping, segments. Each segment is windowed by a sliding time window and next is subjected to spectral analysis using FT. In this approach the window $w(t)$ defines each time segment to be analyzed (figure 2), thus resulting in a two-dimensional function STFT $X(t, f)$ defined by [28]:

$$
X(t, f)=\int_{-\infty}^{\infty} x(\tau) w(\tau-t) e^{-j f \tau} d \tau
$$

In the simplest case, the window $w(t)$ may be a square function, however, generally other types of windows, e.f. Hnning window are used.

The spectrogram $S_{x x}(t, f)$ of signal $x(t)$ is obtained by computing the squared magnitude of (5):

$$
S_{x x}(t, f)=|X(t, f)|^{2}
$$


The spectrogram is a real-valued, nonnegative distribution which provides a signal representation in the time-frequency domain.

Graphs of spectrograms obtained using STFT for the beginning of signals BUB601, BUB401 and BUB101 are shown in figure 3 .
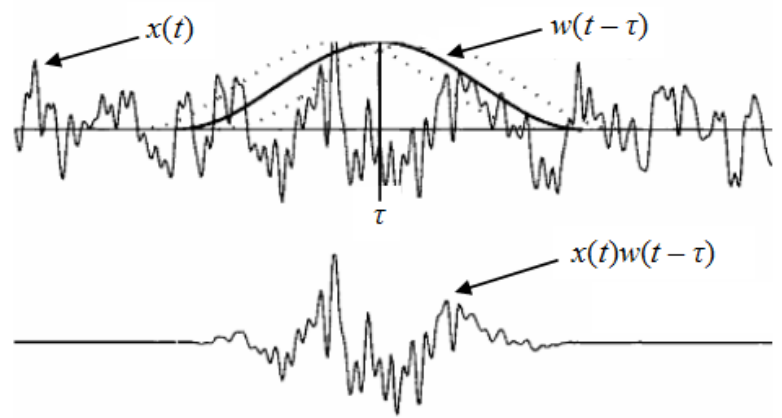

Figure 2. Application of sliding time window to $x(t)$ signal [28].
In the analyses the following settings determined experimentally were used: segment width of 10,000 samples, Hamming window, $80 \%$ overlapping.

It is easy to note that a spectrogram for three analyzed signals differ significantly. However, numerical parameters which allow separation of the classes (flow structures) were required. On the basis of many experiments carried out it was found that the following features may be proposed:

- maximum value of the sum of the spectrogram amplitudes above a fixed amplitude threshold $A g=$ 0.2. Graphs of such sums are shown on the right side of spectrograms in figure 3 ("Spectrogram sum"),

- area under the graph of "Spectrogram sum" for the first five components (frequency range from 0 to $f g=$ $5 \mathrm{~Hz}$ ). The value of $f g=5 \mathrm{~Hz}$ was determined experimentally.
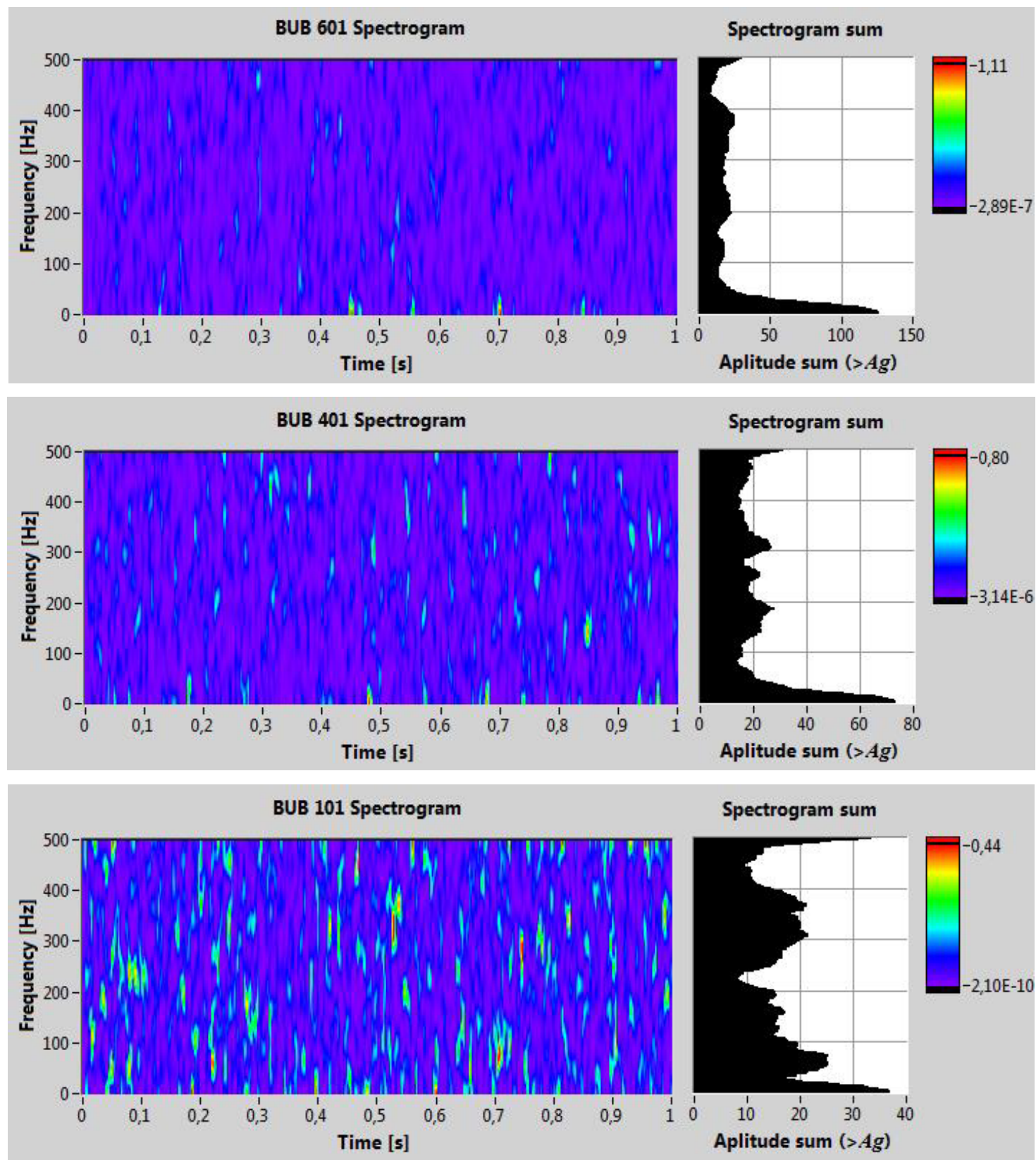

Figure 3. Spectrograms determined for signals BUB601, BUB401 and BUB101 using STFT. 
- area under the graph of spectrogram sum in the frequency range from 0 to $f g=5 \mathrm{~Hz}$, divided by the total area.

The graphs presented in figure 4 show the values of the mentioned above features as a function of the segment number.
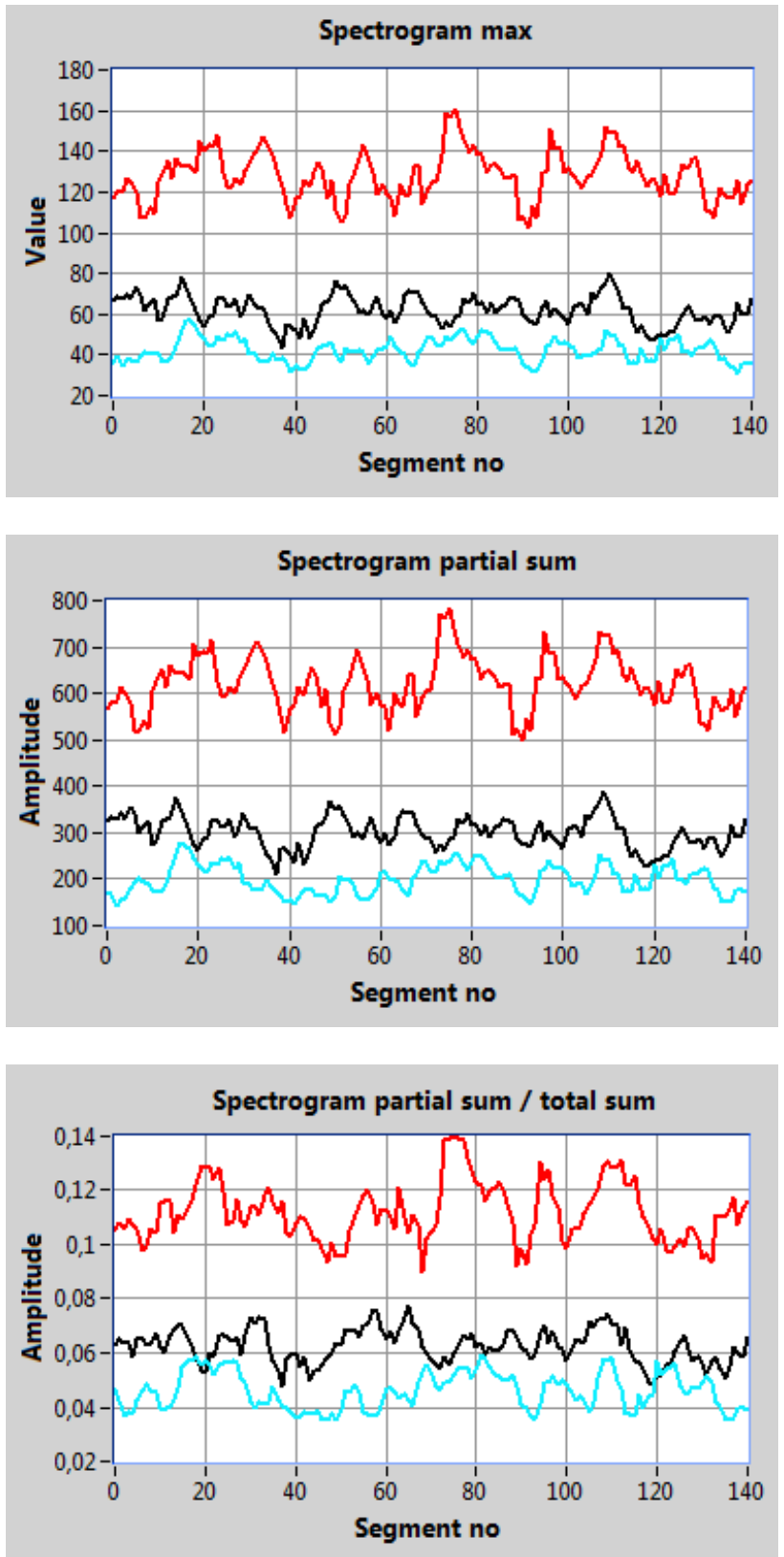

BUB601 - plug flow

BUB401 - transitional plug - bubble flow

BUB101 - bubble flow

Figure 4. Features determined on the basis of STFT spectrograms.

The results presented in figure 1 suggests that the best separation for three analyzed structures of air-water flow have features based on the CSDF magnitude.

The STFT spectrogram may also be used for flow pattern recognition. As can be observed in figure 4 the best separation of patterns represented by signals
BUB601, BUB401 and BUB101 has the maximum value of the sum of STFT spectrogram.

\section{Conclusions}

In this article stochastic signals recorded from scintillation detectors for three structures of water-air flow as plug, bubble, and transitional plug-bubble flows in horizontal pipeline were analyzed in the frequency domain. Based on the obtained results it can be stated that the selected parameters of ADF, CSDF and STFT spectrogram may be useful to the recognition of liquidgas flow pattern.

According to the authors an improvement can be accomplished using the magnitude of the cross-spectral density, rather than the autospectral density function. On the basis of the carried out experiments, it was found that the best separation of the patterns give: maximum of the $|\mathrm{CSDF}|$ and relative area under the $|\mathrm{CSDF}|$ graph of recorded signals.

In the case of the STFT spectrogram the best separation for considered classes of flow has area under the graph of spectrogram sum in the selected frequency range of the transform.

It can be concluded, that the mentioned above features determined in the frequency domain would be applied to liquid-gas flow pattern recognition using machine learning methods.

\section{References}

1. S. Blaney, H. Yeung, Flow Meas. Instrum. 19, $57-$ 66 (2008)

2. O. Arjmandi-Tash, A. Zarghami, R. Zarghami, Particul. Sci. Technol. 32, 498-505 (2014)

3. Y. Chen, et al, Chem. Eng. Sci. 129,156-167 (2015)

4. F. Johnsson, Int. J. Multiphas. Flow 26, 663-715 (2000)

5. C.M. Salgado, Prog. Nucl. Energy 52, 555-562 (2010)

6. Z. Sun, H. Zhang, Meas. Sci. Technol. 19, 015401 (2008)

7. G.H. Roshani, Measurement 62, 25-32 (2015)

8. C.M. Salgado, Appl. Radiat. Isot. 67, 1812-1818 (2009)

9. M. Khorsandi, et al, Radiat. Meas. 59, 183-187 (2013)

10. H. Ding, et al, Flow Meas. Instrum. 18, 37-46 (2007)

11. T. Elperin, M. Klochko, Exp. Fluids 32, 674-682 (2002)

12. E.S. Rosa, et al, Int. J. Multiphas. Flow 36, 738-754 (2010)

13. J.S. Bendat, A.G. Piersol, Random data - analysis and measurement procedures (John Wiley, New York, 2010)

14. J.H. McClellan, R.W. Schafer, M. A. Yoder, Signal processing first (Prentice Hall, New Jersey 2003) 
15. A.V. Oppenheim, R.W. Schafer, J.R. Buck, Discrete -Time Signal Processing (Prentice Hall, New Jersey 1999)

16. R. Hanus, Int. J. Multiphas. Flow 72, 210-217 (2015)

17. M. Zych, et al., EPJ WoC 92, 02122 (2015)

18. R. Hanus, et al, JPCS 530, 012042 (2014)

19. M. Zych, et al., MATEC WoC 18, 02004 (2014)

20. M.S. Beck, A. Plaskowski, Cross correlation flowmeters - their design and application (Adam Hilger, Bristol, 1987)

21. P. Hanus, MS thesis, AGH University of Science and Technology, Krakow 2014 (in Polish)
22. M. Zych, et al, Flow Meas. Instrum. 35, 11-15 (2014)

23. R. Hanus, L. Petryka, M. Zych, Flow Meas. Instrum. 40, 58-63 (2014)

24. L. Petryka, et al, EPJ WoC 92, 02065 (2015)

25. M. Zych, et al., EPJ WoC 92, 02121 (2015)

26. R. Hanus, et al., Math. Probl. Eng. 2014, 475735 (2014)

27. L. Liebenberg, J.P. Meyer, Exp Therm Fluid Sci. 31, 127-140 (2006)

28. L. Sörnmo, P. Laguna, Bioelectrical signal processing in cardiac and neurological applications (Elsevier Academic Press, San Diego, 2005) 